\title{
La problématisation historienne au lycée : articuler les échelles de lecture d'un document sur les Guerres de Vendée
}

Historian problematization in high school: associating scales of reading a document about the Vendée Wars

\section{Lucie Gomes}

\section{OpenEdition Journals}

Édition électronique

URL : https://journals.openedition.org/educationdidactique/5126

DOI : 10.4000/educationdidactique.5126

ISSN : 2111-4838

\section{Éditeur}

Presses universitaires de Rennes

\section{Édition imprimée}

Date de publication : 30 décembre 2019

Pagination : 109-125

ISBN : 978-2-7535-8041-1

ISSN : $1956-3485$

\section{Référence électronique}

Lucie Gomes, «La problématisation historienne au lycée : articuler les échelles de lecture d'un document sur les Guerres de Vendée », Éducation et didactique [En ligne], 13-3 | 2019, mis en ligne le 03 janvier 2021, consulté le 12 octobre 2022. URL : http://journals.openedition.org/educationdidactique/ 5126 ; DOI : https://doi.org/10.4000/educationdidactique.5126 


\section{LA PROBLÉMATISATION HISTORIENNE AU LYCÉE : ARTICULER LES ÉCHELLES DE LECTURE D'UN DOCUMENT SUR LES GUERRES DE VENDÉE}

Lucie Gomes

CREN, Nantes

Dans une recherche empirique en classe d'histoire au lycée, nous testons une séquence forcée pour que les élèves problématisent et construisent du savoir nouveau sur une thématique mémorielle : les Guerres de Vendée. Sans opposer histoire et mémoire, les élèves doivent élucider les raisons des discours sur cet évènement. Larticulation entre l'échelle micro (les informations dans le document) et l'échelle macro (auteur, date, nature) rend possible le dépassement du registre " ethnologique » issu des récits mémoriels locaux pour imaginer d'autres lectures sur cet événement.

Mots-clés : histoire et mémoire, didactique de l'histoire, problématisation, étude des sources, historiographie.

Historian problematization in high school: associating scales of reading a document about the Vendée Wars

In an empirical research in high school history class, we have tested a "forced sequence " in order for students to problematize and to build new knowledge on a memorial subject prone to social controversies and historical debates: the Vendée Wars. Without opposing history and memory, students must elucidate the reasons for writing about this event. The articulation between the micro scale (information in the document) and the macro scale (author, date, kind of document) makes it possible to go beyond the "ethnological" register from local memory stories to imagine other readings on this event.

Keywords : history and memory, didactics of history, problematization, study of sources, historiography. 


\section{UNE ÉTUDE SUR LA PROBLÉMATISATION EN HISTOIRE}

L'étude présentée ici s'appuie sur une recherche plus large sur l'apprentissage de la problématisation par les élèves en lycée. Ce cadre théorique est utilisé en didactiques pour comprendre la façon dont les élèves accèdent à des savoirs problématisés : des savoirs où l'élaboration des problèmes est privilégiée sur la recherche de solutions. Fabre (2017) et Orange (2005) expliquent le processus par lequel des savoirs problématisés se construisent : la mise en tension entre un registre empirique (des données) et un registre des modèles explicatifs (les idées explicatives sur le problème) peut produire du savoir nouveau, sur la base du savoir déjà-là des élèves et des éléments fournis par le dispositif. Ce savoir nouveau se situe parfois dans un registre explicatif différent de celui dans lequel les élèves se trouvent initialement, tel un changement de paradigme scientifique. Des séquences forcées (Orange 2010), basées sur le débat et la réflexivité sur le problème en construction, permettent aux didacticiens qui utilisent ce cadre de comprendre à quelles conditions cette construction des savoirs est possible pour les élèves, en référence au processus de problématisation que connaissent les scientifiques dans la discipline concernée.

En histoire, la problématisation est spécifique à l'étude du passé. Nous décidons de nous appuyer sur l'épistémologie des historiens autour du travail sur les sources : la pensée historienne qu'ils déploient est éloignée du réalisme constaté chez les élèves. Audigier (1992 et 1995) a ainsi développé la façon dont les élèves considèrent le document venant du passé : il est transparent et il dit la vérité, en extraire des informations suffirait donc au travail en histoire :

Le document subit un traitement très « positiviste »; il est lu et expliqué au premier degré, c'est-à-dire que le jeu des questions qui lui sont posées et des réponses attendues, en fait l'expression, la représentation, le récit... de la réalité. (Audigier 1992)

L'objectif n'est pas de faire des élèves des petits historiens, mais peuvent-ils néanmoins dépasser ce réalisme en direction d'un usage critique des sources, et construire des problèmes historiques au lycée? Notre hypothèse est que le dépassement du réalisme des sources est central pour problématiser. La problé- matisation, qui passe par l'exploration des possibles, nous sert donc à la fois pour monter une séquence forcée favorisant la mise en tension entre les données et modèles explicatifs, et comme cadre d'analyse de l'activité de l'élève pour comprendre ce qui permet la construction du savoir nouveau.

\section{UNE ÉTUDE SUR UNE THÉMATIQUE MÉMORIELLE}

Dans cette étude de cas, nous nous intéressons plus particulièrement à une problématisation où le savoir déjà-là des élèves a été influencé par des dispositifs mémoriels comme un parc touristique fournissant une explication globale sur un évènement. En effet, le Puy du Fou est fortement fréquenté par les jeunes Vendéens et il diffuse un récit mémoriel, notamment sur les Guerres de Vendée. La valorisation de la monarchie et de la chrétienté est présente dans de nombreux spectacles et le Vendéen y est essentialisé comme un peuple aux valeurs conservatrices, différentes de celles du reste des Français. La mémoire victimaire sur les Guerres de Vendée est entretenue sur ce site, comme dans d'autres espaces touristiques dont l'Historial et le Mémorial de Vendée ou encore La Chabotterie. Quand mémoire et histoire viennent à se contredire, comment penser la construction du savoir en classe? Létourneau et Moisan (2004) qui étudient la situation québécoise, Tutiaux-Guillon (2008) celle en France ou Fink (2014) en Suisse nous apprennent la grande résistance des explications des élèves sur les thématiques mémorielles. Ces explications collectives du passé établissent un récit sur le passé cohérent bien qu'éloigné des travaux des historiens. Il est donc difficile de se départir de tels récits dans le travail en classe d'histoire.

Loin d'être pauvre et incohérente, cette vision est en effet ficelée et structurée. Elle témoigne de l'assimilation ample d'un récit de l'aventure québécoise - celui du «peuple abandonné, reclus, se redressant mais toujours hésitant à s'accomplir »-(..) alors même que ses assises, tant scientifiques que politiques, sont ébranlées par les avancées de la recherche historienne. (Létourneau, Moisan 2004, p. 325).

Les questions mémorielles posent soucis pour les enseignants qui, après avoir occulté pendant de nombreuses années ces thématiques, se retrouvent 
au cour d'une demande sociale pour les traiter. Tutiaux-Guillon (2008) explique cette difficulté :

En outre, même si les enjeux de la mémoire ne sont pas étrangers à la recherche historique, le travail de l'historien ne peut, en bonne déontologie, être orienté par le devoir de mémoire. La confrontation entre histoire et mémoire est donc devenue critique et conflictuelle. De là, le malaise d'enseignants qui enracinent leur identité professionnelle et la légitimité de leur discipline dans l'histoire savante et se trouvent confrontés à l'injonction mémorielle.

En Vendée, les élèves grandissent au contact d'une mémoire construite depuis longtemps. L'enquête conséquente réalisée par Martin et Suaud (1996) démontre la création de cette identité vendéenne par des spectacles vantant le christianisme et la ruralité qui seraient spécifiques à ce territoire. Le discours du professeur porte peu dans ces cas-là, tel un savoir désincarné, détaché du contexte de sa production, en concurrence avec un récit répété de façon ludique dans les sites touristiques. Cela fait de ces thématiques mémorielles des questions sensibles avec des savoirs potentiellement refusés par les élèves. Pourtant, ces mémoires construites inspirent les historiens qui prennent aujourd'hui comme objet de recherche la construction des identités. La problématisation peut alors être utile pour la construction du savoir si on considère qu'il ne s'agit pas de fournir aux élèves un récit contradictoire au leur, mais de leur permettre de construire un problème dont la fonction est de questionner le récit traditionnel lorsqu'il est confronté, par l'enquête historienne, aux sources.

Les deux discours, celui issu du savoir historique et celui venant de la doxa royaliste, peuvent apparaître aussi légitimes l'un que l'autre pour les élèves. Nous risquerions alors le relativisme face au passé en voulant leur imposer une vérité non questionnée. Le savoir problématisé n'est pas une explication alternative au récit initial dominant, il réside dans les raisons de passer d'un récit à un autre, élaborées au cours de la problématisation. C'est donc la logique politique de la construction mémorielle qui est alors interrogée. Des études de cas montrant l'intérêt de la construction d'un savoir problématisé ont ainsi été réalisées à plusieurs niveaux de classe et sur des thématiques diverses comme les relations seigneurs/ paysans au Moyen Âge en fin de primaire (Le Marec, Doussot \& Vézier, 2009), la chute de la monarchie pendant la Révolution française (Doussot 2010) ou encore sur l'expérience de guerre à travers les lettres d'un soldat durant la Première Guerre mondiale (Doussot \& Vézier 2014). C'est en référence à ces travaux et notamment la thèse de Doussot (2011) que nous avons pu élaborer et analyser cette séquence.

\section{L'HYPOTHÈSE DE L'ARTICULATION DES ÉCHELLES DU DOCUMENT POUR FAIRE DE L'HISTOIRE}

Ces études ainsi que d'autres recherches didactiques (Tutiaux-Guillon, 2008; Cariou, 2012) montrent que les activités que l'on fait habituellement faire aux élèves en classe d'histoire consistent à :

- Présenter le document avec les données contextuelles de celui-ci : nous l'appellerons l'échelle macro puisqu'il s'agit de nommer l'auteur, la date et la nature du document, et ce qui constitue le contexte de la source étudiée.

- Prélever des informations du document : nous l'appellerons l'échelle micro du document puisque cela consiste à se focaliser sur des aspects précis du contenu.

Cependant, c'est selon nous par l'articulation des modèles explicatifs de l'auteur du document (présents à l'échelle micro) avec la critique de celuici (échelle macro) que les élèves peuvent accéder à certains problèmes historiques. C'est par exemple le cas lorsque, au cœur d'une discussion sur la violence des combats dans les tranchées, l'enseignante introduit la question de ce qu'en garde la lettre du soldat étudié : des élèves, immédiatement, mettent en doute le fait que "le soldat racontait tout dans sa lettre » sur la base du fait que constitue la transmission de nouvelles à sa famille dans une telle situation. Le soldat ne dit sans doute pas tout, il tient à préserver ses proches (Doussot \& Vézier, 2014, p. 131). Si on se réfère à la pratique des historiens et à ce que dit Bloch par exemple, c'est dans l'articulation entre la critique de la source et le problème qui est posé que se construit le savoir historique :

Beaucoup de personnes et même, semble-t-il, certains auteurs de manuels se font de la démarche de notre travail une image étonnamment candide. $\mathrm{Au}$ commencement, diraient-ils volontiers, sont les documents. L'historien les rassemble, les lit, s'efforce 
d'en peser l'authenticité et la véracité. Après quoi et après quoi seulement, il les met en œuvre... Il n'y a qu'un malheur : aucun historien, jamais, n'a procédé ainsi. Même lorsque, d'aventure, il s'imagine le faire (Bloch, 1997, p. 77).

Aujourd'hui pourtant, c'est souvent ainsi que sont organisés les exercices sur les documents en classe d'histoire. Les deux étapes («identifier des documents » et « critiquer des documents» dans le BO $\mathrm{n}^{\circ} 4$ d'avril 2010 sur les programmes de Seconde) sont successives et l'articulation n'en est pas pensée dans le travail à réaliser par les élèves. Nous postulons, sur ces bases didactiques et épistémologiques, que cette articulation s'apprend par l'articulation des échelles entre micro (les informations du document) et macro (les données contextuelles du document), des allers-retours dans le cadre d'une enquête menée par les élèves.

\section{UNE ÉTUDE SUR L'ENSEIGNEMENT DES GUERRES DE VENDÉE, AVEC LE REGISTRE ETHNOLOGIQUE COMME OBSTACLE}

Nous décidons d'expérimenter un travail autour des enjeux mémoriels et de liaisons entre critique de la source et interprétation de celle-ci sur les Guerres de Vendée, dans le chapitre consacré à la Révolution française en classe de Seconde. Les élèves habitent en Vendée et côtoient une mémoire entretenue sur plusieurs sites touristiques dits historiques (le Puy du Fou, l'Historial et le Mémorial de la Vendée, la forêt de Grasla, la Chabotterie). Les logiques narratives de ces lieux peuvent induire chez les élèves des explications où le Vendéen est un homme bien différent culturellement du reste des Français, plus conservateur et plus chrétien, ce qui aurait causé sa rébellion. La difficulté qui se présente est celle d'opposer deux récits contradictoires : le discours de la science historique problématisée et le discours mémoriel construit par les acteurs des sites touristiques du département. Il s'agirait alors de construire le problème à la façon d'une enquête comme le développe Fabre dans son étude sur Dewey (2015). Il faudrait remonter à la production des récits mémoriels construits dans les explications des élèves par les discours portés dans les parcs, en mettant en discussion leurs fondements, plutôt que de réfléchir directement sur l'évènement lui-même.
Ici, le récit mémoriel des sites touristiques en Vendée s'appuie sur un présupposé ethnologique d'existence du peuple vendéen. Nous faisons le parallèle avec ce qu'explique Bazin (2008) sur le peuple Bambara. En effet, l'invention de l'ethnie commence en premier lieu comme une procédure d'identification. Le « paradigme ethnologique » consisterait à spécifier les cultures des groupes humains et à les essentialiser. Les caractéristiques culturelles alors attribuées à ce groupe constituent pour les membres de ce groupe la façon d'expliquer leur comportement qu'ils considèrent comme une identité culturelle. Les Vendéens n'ayant pas de singularité culturelle au moment de la Révolution, la constitution d'une mémoire autour des Guerres de Vendée en fait l'acte fondateur de création d'une « ethnie » dans le sens qui est critiqué par Bazin. Nous nous appuyons aussi pour cela sur Chartier (2000), qui en réfléchissant aux origines culturelles de la Révolution française explique qu'on reconstruit a posteriori un évènement (ici ce sont les Guerres de Vendée) au regard du récit qu'on souhaite avoir sur celui-ci (qui diffère selon les acteurs, les périodes), le rendant inévitable et compréhensible dans une logique cause-conséquence. On retrouve dans le récit mémoriel vendéen des reconstructions comparables essentialisant le peuple de Vendée pour mieux expliquer sa rébellion. Pourtant, les sciences sociales ont comme principe de questionner les catégories sociales naturalisées. Remettre en question l'existence d'un peuple vendéen peut ainsi permettre de sortir de ce paradigme « ethnologique » (Bazin, 2008) auquel les élèves ont été confrontés très souvent pour interroger autrement le passé.

Dans la présentation, nous expliquerons d'abord comment nous avons conçu une séquence dans un objectif de problématisation. Ensuite, nous analyserons comment les élèves entrent dans cette étude dans une articulation d'échelles que nous estimons nécessaire à une problématisation mobilisant la pensée historienne sur le travail des sources.

\section{CONSTRUIRE UNE SÉQUENCE FORCÉE POUR QUE LES ÉLÈVES PROBLÉMATISENT}

\section{Se référer aux débats sur une question socialement vive}

Afin d'identifier le problème spécifiquement historique à faire construire dans la classe, nous 
nous tournons du côté de la discipline de référence pour faire le point sur les débats historiographiques sur le sujet et identifier ensuite comment l'approche didactique peut s'en saisir. Ce qui fait problème dans cet épisode de l'histoire de la Révolution en Vendée, ce ne sont pas les faits : l'accaparement des biens nationaux par la bourgeoisie urbaine, la constitution civile du clergé qui créé le clergé assermenté face aux prêtres réfractaires qui refusent de prêter serment, la levée des 300000 hommes pour conduire l'armée contre l'ennemi de l'extérieur... Ceci conduit de nombreux paysans français à se révolter. Le rapport Barrère de 1793 à la Convention demande la répression de ces ennemis de l'intérieur et les généraux appliquent cet ordre en commettant des crimes de guerre contre des civils victimes des massacres. La polémique est liée aux interprétations sur la période. Or en histoire les interprétations ne découlent pas directement des données, mais il existe un lien étroit entre les idées explicatives sur une thématique et les données sollicitées parmi les traces possibles pour établir des faits. Cette imbrication forte entre ce qu'on choisit de prélever dans les sources et les idées explicatives qu'on tient pour vraies s'inscrit dans un paradigme spécifique qui fonde la légitimité des explications proposées tout en induisant une exploration systématique, car délimitée, des données et des idées. Autrement dit, le paradigme en histoire permet l'enquête en délimitant les questions possibles et en obligeant l'exploration dans ce cadre. Mais quand l'objectif est idéologique, considérer les Vendéens comme un peuple dans une logique ethnologique par exemple, l'enquête tend à se réduire à une recherche rhétorique des idées et des données conformes au présupposé. Martin (1992 et 2000) s'est intéressé au débat historiographique relancé dans les années 1980 avec le bicentenaire de la révolution. La thèse de Sécher (1986) sur un génocide vendéen (hypothèse ethnologique défendue dans une thèse polémique, expliquant que la République a organisé une extermination du "peuple vendéen » en réponse aux Guerres de Vendée) vient rencontrer les récits mémoriels construits par des politiques, soutenus et sollicités par des polémistes conservateurs (Martin 1992). À la suite d'une récompense reçue par Sécher, Martin, qui a toujours nié les conclusions de ce dernier, réitère les critiques sur les conclusions génocidaires :
Poser une bonne question ne préjuge pas de la valeur de la réponse. Il était nécessaire, je l'ai dit à maintes reprises, de se demander si la guerre de Vendée avait été un génocide. Mais les critères qui définissent un pareil crime, à commencer par la désignation précise d'une population visée, manquent. [...] Où est le génocide? (Martin in Causeur 24/10/12 $2^{1}$.

Le récent ouvrage de Buisson (2017) préfacé par de Villiers reprend les théories sur le génocide vendéen. L'utilisation de l'histoire se fait alors à des fins politiques et sociétales pour servir les idées conservatrices sur le plan politique de ces deux hommes : le Vendéen devient le symbole de l'homme aux vraies valeurs, victime du passé, attaché à la religion et opposé à certains aspects de la république. La polémique touche donc à la fois le monde scientifique et la société, ce qui ne facilite pas un travail serein et apaisé sur le sujet, et encore moins en classe d'histoire. Ce sujet d'étude fait donc partie des questions socialement vives (Legardez \& Simonneaux 2006) lorsque cet enseignement se fait en Vendée puisque cette thématique correspond aux trois niveaux de vivacités : c'est un objet de controverse dans la société mais aussi dans le débat scientifique avec des répercussions à l'école. Les élèves arrivent en classe d'histoire avec des connaissances sur le sujet et cela demande un traitement spécifique par les enseignants devant tenir compte des explications des élèves sur ces thématiques.

Les guerres de Vendée ne sont des objets de la recherche historique aussi importants que parce qu'elles sont réutilisées à des fins mémorielles à divers moments de l'histoire. Martin dit ainsi que cette " querelle pourrait bien être l'un des derniers épisodes de la "guerre civile historiographique" que se livrent les Français depuis 1814. " Il y a donc continuité entre le combat lui-même qui a dès le début une dimension mémorielle et la question actuelle de la connaissance de l'évènement. Histoire et mémoire, loin d'être opposées sont ici entremêlées. Ainsi, nous reprenons les catégorisations historiographiques de Prost et Winter (2004) où les auteurs sur la première guerre mondiale distinguent une historiographie politique, une économique et sociale, et une culturelle. Concernant les Guerres de Vendée, nous identifions un paradigme répandu sur cette thématique : nous pouvons considérer que les polémistes conservateurs s'appuient sur une logique ethnologique. Nous estimons qu'il s'agit d'un para- 
digme puisque cela tient ensemble leurs explications et les données qu'ils sélectionnent. En problématisation, nous appelons cela le registre explicatif. C'est ce principe de classification ethnologique qui peut être discuté dans une perspective historique puisque les élèves en Vendée y sont soumis par leurs visites touristiques.

\section{Du débat sociétal au problème à construire en classe}

Le débat sociétal est donc l'affrontement de deux récits irréconciliables sur le passé : l'un caractérisant une répression dans le cadre d'une révolte, l'autre construisant une mémoire autour d'un génocide de la nation contre un peuple différent de celle-ci. Il s'agit pour nous de repenser en problématisation (Le Marec, Doussot, Vézier 2009), l'enseignement de l'histoire en considérant qu'on problématise de façon spécifique à cette discipline. Ginzburg explique cela dans la préface de l'ouvrage de Zemon Davis (2008, p. 33-34) :

Le principe de réalité et d'idéologie, le contrôle philologique et la projection dans le passé des problèmes du présent s'entrecroisent, se conditionnent réciproquement, à tous les instants du travail historiographique, sans exception - de l'identification de l'objet à la sélection des documents, aux méthodes de recherche, aux critères de preuve, à la présentation littéraire. L'enquête historienne se distingue donc par ce travail des sources « à tous les instants » considérées comme un acte dans un contexte passé.

C'est ce qui distingue précisément le travail problématisé des historiens de celui des polémistes: ces derniers, pour construire un récit cohérent avec leur idéologie, vont chercher des informations dans les documents du passé, mais ils évitent de faire une exploration systématique des solutions possibles qui permettrait d'évaluer telle ou telle argumentation singulière; autrement dit, ils jouent sur l'échelle micro (les informations dans le document) sans jamais la contrôler par l'échelle macro (les données contextuelles du document). Mais qu'en est-il quand le sujet traité porte sur une question mémorielle pour les élèves? Peuvent-ils entrer dans le processus de problématisation et construire un savoir nouveau sur le passé? Fink (2014, p. 12) montre les difficultés des enseignants à se saisir des savoirs controversés, elle introduit ainsi son ouvrage :

Tandis que les élèves sont au contact de multiples "vérités » qui circulent dans l'espace public et médiatique, les enseignants effectuent des choix par rapport aux savoirs qu'ils transmettent et à la manière dont ils le font. (...) Lécole est alors un espace fermé sur lui-même. Mais l'école peut aussi s'ouvrir aux enjeux de société en se pensant comme un lieu de réflexion, où les enseignants et les élèves s'interrogent ensemble sur la manière de restituer de la complexité.

Nous faisons l'hypothèse que la problématisation peut permettre de construire des savoirs en s'interrogeant sur les intentions de ceux qui construisent une mémoire plutôt que de l'attaquer de façon frontale par un récit alternatif " prouvé » avec des documents contradictoires. Cela serait d'ailleurs contre-productif, fournissant aux élèves deux récits vus comme vrais et développant chez eux le relativisme sur le passé. Ce travail sur les intentions permet une pensée historienne qui lie critique de la source et interprétation, redonnant toute sa place à la recherche des conditions du problème, au questionnement plutôt qu'à la solution. Pour arriver à ces fins, une séquence forcée est donc établie dans laquelle les élèves seront soumis à des récits subjectifs sur les Guerres de Vendée. En effet, ce type d'étude didactique développée par Orange (2010) a l'avantage de nous permettre de tester un dispositif favorisant la problématisation des élèves par une réflexion sur celle-ci en amont, au cours et après la séquence. Proches de l'ingénierie didactique, les séquences forcées s'en distinguent par la co-élaboration et la co-mise en œuvre entre didacticien et enseignant, mais chacun a une responsabilité spécifique dans le but de produire des phénomènes didactiques pertinents pour la recherche. L'enseignant enseigne mais participe au réajustement entre chaque séance :

Les situations forcées sont des situations d'enseignement qui ne sont généralement pas isolées, mais organisées en une séquence d'enseignement (d'où la notion de séquences forcées); elles sont construites au sein d'un groupe de recherche comportant des chercheurs en didactique et des enseignants experts. L'ensemble du groupe connaît le cadre théorique et la problématique de la recherche. 
La séquence en question est définie par des objectifs pédagogiques (quels apprentissages?) et des objectifs de recherche (que veut-on construire et/ou observer). Les objectifs pédagogiques incluent les objectifs que l'on fixe habituellement et/ou institutionnellement pour la classe, mais ils prennent aussi en compte le cadre théorique de la recherche. Ils sont définis par le groupe; ils sont contrôlés par les enseignants. Les objectifs de recherche sont définis par le groupe; ils sont contrôlés par les chercheurs didacticiens (Orange 2010, p. 77).

Nous sommes ici la chercheuse et la professeure, ce qui, bien que cela puisse présenter un biais dans la recherche, apporte un avantage réel dans la mise en œuvre au cours de la recherche. Nous focaliserons notre attention sur ce qui a été le plus pertinent pour les élèves dans la séquence pour problématiser : le récit d'une noble vendéenne, écrit trente ans après les faits et sur lequel les élèves doivent se questionner davantage sur les raisons de production d'une telle trace historique que sur le contenu. La séquence se compose de deux séances. Ils doivent d'abord en groupe élaborer une hypothèse concernant les regards portés sur les Guerres de Vendée à partir des sites touristiques. Cela permet d'entrer dans la question des récits produits sur un évènement ce qui sera utile pour les savoirs visés ultérieurement. Ensuite les élèves étudient quatre documents ${ }^{2}$ avec un tableau à remplir. Ces documents sont postérieurs aux Guerres de Vendée et ont été choisis car ils portent des intentions de reconstruction du passé : des témoignages de personnes ayant vécu l'évènement ou des constructions mémorielles tardives. Le tableau est construit pour permettre l'articulation entre données et idées explicatives afin que les élèves n'en restent pas aux pratiques habituelles en classe d'histoire. La séquence forcée a pour principe d'être réélaborée en cours de travail pour prendre en charge l'incertitude des productions des élèves et les engager dans un travail réflexif explicite : c'est ce que nous faisons lors de la deuxième séance en soumettant aux élèves ce qu'ont dit certains de leurs camarades sur ces documents et ils doivent alors compléter leur analyse avec des éléments qu'ils ont laissé de côté. Ils doivent ensuite rédiger une synthèse en groupe et terminer par un questionnement sur les récits produits par les sites touristiques.

Il s'agit par ce dispositif de permettre aux élèves une réflexivité sur le travail en cours par une variation des échelles de lecture des documents. Ils doivent passer ainsi de leur analyse micro (les informations dans le document), à leur analyse macro (les données contextuelles du document) pour aller vers une articulation des échelles de lecture du document. C'est ce phénomène didactique que nous avons souhaité produire dans cette étude pour mesurer sa pertinence dans la construction de savoirs problématisés.

\section{LA PROBLÉMATISATION EN HISTOIRE : ARTICULATION DES ÉCHELLES ENTRE MICRO ET MACRO}

\section{Un registre explicatif ethnologique}

Après avoir fait discuter les élèves sur le regard porté par les sites touristiques concernant les Guerres de Vendée, nous leur demandons de passer de leurs conclusions sur le tourisme à une hypothèse sur les regards qui peuvent être portés sur ces guerres. Ils n'ont pas encore eu accès aux documents. Le groupe étudié, quatre élèves de la classe filmés en continu, redéfinit la consigne donnée et ils cherchent à savoir ce que voulaient les Vendéens en se rebellant. Pour l'article, tous les échanges ne sont pas retranscrits mais nous sélectionnons les tours de parole qui font avancer la réflexion entre les élèves. Voici de quoi débat le groupe ${ }^{3}$ avant d'avoir eu accès aux documents :

Transcription de la séance 1 , étape 1

\begin{tabular}{|l|l|l|}
\hline 40 & A & En fait ils étaient contre la Révolution. \\
\hline 41 & M & Contre la démocratie. \\
\hline 42 & E & $\begin{array}{l}\text { Non mais c'était la République, c'était pas tout de suite la démocratie. De toute façon, ils savaient pas ce que } \\
\text { c'était, ils pouvaient pas être contre tout de suite. À l'époque ils étaient très chrétiens. }\end{array}$ \\
\hline 43 & $\mathrm{~J}$ & Ils étaient contre, ils voulaient remettre le roi.
\end{tabular}




\begin{tabular}{|c|c|c|}
\hline 44 & E & Toujours maintenant. \\
\hline 45 & $\mathrm{~J}$ & Oui mais pour eux le roi c'était le descendant de Dieu. \\
\hline 46 & E & Ah ouais? \\
\hline 47 & M & Ouais c'était euh un saint quoi. \\
\hline 48 & $\mathrm{~J}$ & On cherche à expliquer mais on mélange... \\
\hline 49 & M & Du coup, pour l'hypothèse. \\
\hline 50 & A & Les Vendéens étaient totalement opposés à cette Révolution et ils étaient pour l'Ancien Régime. \\
\hline 51 & E & Ce qu'était la monarchie absolue. \\
\hline 52 & M & Tu peux répéter parce que là... \\
\hline 53 & E & Alors, les Vendéens... \\
\hline 54 & M & Étaient pour la monarchie absolue. \\
\hline 55 & $\mathrm{~J}$ & Pas la monarchie absolue mais ils voulaient au moins le roi. \\
\hline 56 & A & Et pour l'Ancien Régime. \\
\hline 57 & $\mathrm{~J}$ & Parce que ça m'étonnerait qu'ils soient pour la monarchie absolue. \\
\hline 58 & A & Monarchie ou monarchie absolue? \\
\hline 59 & $\mathrm{~J}$ & Monarchie, ils voulaient pas la monarchie absolue, ils voulaient juste la monarchie. \\
\hline 60 & M & Donc ils voulaient un roi, mais quand même avoir des pouvoirs. \\
\hline 61 & $\mathrm{~J}$ & Oui. De toute façon, personne aimait la monarchie absolue. \\
\hline 62 & E & Mais si ça se trouve, y'a des gens qui aimaient bien la monarchie absolue hein. \\
\hline 63 & M & Ben, en fait le fait de ne rien avoir à décider... \\
\hline 64 & E & $\begin{array}{l}\text { Oui mais vu qu'ils ne connaissaient rien d'autre... Tu veux pas quelque chose si tu connais rien d'autre. Quand } \\
\text { on nous parle de monarchie absolue maintenant, on se dit quoi? Ben, c'est pareil pour la République et tout. }\end{array}$ \\
\hline 65 & $\mathrm{~J}$ & Oui ils n'y connaissaient rien à l'époque. \\
\hline 66 & E & Non et puis ils n'allaient pas tous à l'école. \\
\hline
\end{tabular}

Ce qui nous semble intéressant ici, c'est que les élèves perçoivent la situation de la population vendéenne de l'époque comme spécifique à ce territoire. En effet, à plusieurs reprises, ils désignent les Vendéens comme un tout uniforme (tour de parole 40-43, tdp 50, tdp 53, tdp 55, tdp 57, tdp 60, tdp 66), une nation différente de la nation française, et qui posséderait donc son récit propre où le royalisme du Vendéen est constitutif de sa façon de penser. Pourtant cette identité vendéenne relève bien de la mémoire construite autour d'un mythe : Avezou
(2013) déconstruit ainsi le mythe autour de la Vendée unifiée en expliquant sur quelles valeurs supposées on a essentialisé le Vendéen : plus rural, plus chrétien, plus attaché aux valeurs traditionnelles, ce qui expliquerait la révolte contre la République durant la Révolution. Nous avons identifié chez Moisan et Létourneau (2004), qui ont étudié la pensée des jeunes Québécois sur leur histoire, la même tendance mythologique pour un peuple qui cherche lui aussi des évènements fondateurs où il peut apparaître comme victime pour créer une histoire commune 
(p. 325-326). La comparaison avec de telles études nous permet d'identifier des constantes : les idées explicatives des élèves sur l'histoire ne viennent pas que de l'école mais aussi de ce qui est transmis par les familles et par le patrimoine mis en scène.

Cela nous permet de comprendre l'obstacle épistémologique des élèves sur ces cas de valorisation d'une histoire unifiée et homogénéisée d'un peuple identifiable : ils se situent dans un registre explicatif ethnologique de différenciation du peuple vendéen qui tient ensemble leurs explications et les données qu'ils sélectionnent. Le découpage initial du monde réel est alors à remettre en question (le découpage en « peuples » ou « cultures ») au profit d'un découpage de l'évènement par l'enquête qui est le sens du travail historique, celui d'étudier des évènements, des situations et non des entités préétablies, dans l'objectif de questionner ces dernières. Chartier (2000) théorise cet enjeu comme histoire culturelle du sociale : il précise (p. 292) sa démarche :

La construction des intérêts ou des évènements par le discours est elle-même socialement enracinée et contrainte. Elle dépend des ressources (...) dont peuvent disposer de manière très inégale, les différents acteurs. Elle renvoie donc aux propriétés sociales objectives qui caractérisent chacun des groupes ou des classes qui constituent le monde social.

Les élèves agissent donc comme s'il n'existait pas plusieurs façons de penser la Révolution en étant Vendéen et comme si cette façon de se la représenter ne se retrouvait qu'ici et pas ailleurs. Ils ont reconstruit la question et plutôt que de réfléchir sur les regards portés sur les Guerres de Vendée, ils cherchent à savoir ce qui pousse les Vendéens à être contre la Révolution : «Les Vendéens étaient totalement opposés à cette Révolution et ils étaient pour l'Ancien Régime » (tdp 50). Les élèves ne sont pas d'accord sur ce que cela signifie. Il y a donc des modèles explicatifs sur la situation de révolte des Vendéens (être pour la monarchie (tdp 43), pour la monarchie absolue (tdp 51) ou craindre ce qu'on ne connaît pas (tdp 65) mais pas de questionnement sur la catégorie de ce peuple essentialisé. Comme les élèves supposent les Vendéens tous royalistes, ils pensent à des modèles explicatifs qui ne remettent pas en cause cet état de fait, donc ils se demandent s'ils sont pour la monarchie ou la monarchie absolue.
Tout l'enjeu est alors de permettre la remise en cause de la catégorisation qu'ils opèrent par un dispositif spécifique pour créer du savoir nouveau. En effet, nous postulons que dans ce cas-là, la problématisation consiste à remettre en question des catégories préétablies et non discutées pour les élèves: si les Vendéens ne sont pas considérés comme un peuple aux caractéristiques différentes, l'évènement de révolte et de Guerres de Vendée devra être relu selon d'autres catégories : sociales, politiques...

\section{L'articulation des échelles pour problématiser en histoire}

Dans la deuxième étape de la séquence, les élèves ont à remplir en groupe le tableau suivant avec les documents fournis par la professeure:

\begin{tabular}{|l|l|l|}
\hline $\begin{array}{l}\text { Présentation } \\
\text { du document. }\end{array}$ & $\begin{array}{l}\text { Les guerres de } \\
\text { Vendée, quels } \\
\text { regards portés } \\
\text { sur la } \\
\text { Révolution? }\end{array}$ & $\begin{array}{l}\text { Dans quel objectif a été } \\
\text { produite cette trace de } \\
\text { l'histoire? }\end{array}$ \\
\hline & & \\
\hline
\end{tabular}

Il a été conçu pour permettre la problématisation. La première colonne se rapproche des habitudes en classe d'histoire. Les élèves risquent d'ailleurs de convoquer les méthodes habituelles en classe d'histoire : auteur, nature, date, informations du document. Ils travaillent alors l'échelle macro du document. La deuxième question s'apparente au prélèvement d'information dans le document, comme cela se fait aussi habituellement, ils sont alors à l'échelle micro du document étudié. Ce que nous souhaitons atteindre c'est l'articulation des échelles entre elles dans la colonne 3, par des allers-retours successifs, pour comprendre les éléments sélectionnés en relation avec l'intentionnalité de production de la trace du passé. La nature, l'auteur, la date du document deviennent des nouvelles données à mobiliser et à mettre en relation avec l'échelle micro. Il s'agit donc d'un changement d'échelles au sens épistémologique, c'est-à-dire lié aux pratiques des historiens sur les sources, et pas seulement comme un zoom différent. Cela les amène à se questionner sur les propos de la noble puis sur les motivations qui la pousseraient à donner cette interprétation des faits (échelle macro). Ils retournent alors vers les explica- 
tions fournies par l'auteure (échelle micro : les informations dans le document) pour les comprendre au regard de ces nouvelles analyses. C'est cette réflexivité qui les autorise à proposer leurs propres modèles explicatifs alors qu'ils étaient auparavant contraints par les modèles explicatifs de la noble.

Le document sur lequel les élèves ont le plus problématisé, en articulant les échelles d'analyse, est le suivant (Manuel d'histoire Belin, Seconde, 2010, p. 270): revanche, sa contextualisation, tant en termes de période (la Restauration) que la réflexion autour de son auteure (une noble) peut permettre de reconsidérer ce qui est dit dans le document. L'acte d'écrire des mémoires et de vouloir inscrire une version de ce qu'il s'est passé, si cela est mis en tension avec des modèles explicatifs essentialisant les Vendéens (du registre ethnologique), peut produire de nouveaux modèles explicatifs dans un registre de l'intentionnalité des acteurs de cet évènement.

\section{Une rébellion paysanne}

«Les paysans se révoltèrent du côté de la Bretière; ils se dispersèrent ensuite dans les paroisses voisines et vinrent trouver M. Sapinaud de Bois-Huguet [...]. "Nous vous prenons, lui dirent-ils, pour notre général et vous marcherez à notre tête." Sapinaud essaya de leur faire envisager les malheurs qu'ils allaient attirer sur eux et sur la Vendée. "Mes amis, leur dit-il, c'est le pot de terre contre le pot de fer. Que ferons-nous? Un seul département contre quatre-vingt-deux! Nous allons être écrasés. [...] Croyez-moi, retournez chez vous et ne vous perdez pas inutilement." Ces braves paysans, loin de se rendre à ses raisons, lui remontrèrent qu'ils ne pourraient jamais se soumettre à un gouvernement qui leur avait enlevé leurs prêtres et qui avait emprisonné leur roi. "On nous a trompés, dirent-ils, pourquoi nous envoiet-on des curés constitutionnels? Ce ne sont pas là les prêtres qui ont assisté nos pères à leurs lits de mort et nous n'en voulons pas pour bénir nos enfants." Mon beau-frère ne savait trop quel parti prendre [...] Enfin, voyant leur obstination, il finit par céder, se mit à leur tête et partit le jour même pour les Herbiers.»

Jeanne:Ambroise de Sapinaud de Bois-Huguet, Mémoires, 1824.

Ce document est extrait de mémoires. Celles-ci fleurissent à la Restauration. Ici il s'agit des mémoires d'une femme, noble vendéenne, qui relate dans cet extrait les aventures de Charles Sapinaud, son beaufrère. Nous sommes trente ans après les faits, et on peut supposer qu'il s'agit d'une entreprise d'héroïsation et de justification. L'auteure essaie de montrer que l'initiative ne relève pas de son beau-frère mais que c'est contraint par les paysans qu'il cède à leurs demandes. Elle vise donc en écrivant une histoire de l'insurrection à montrer que c'est le peuple qui attendait des nobles qu'ils le guident. Les nobles ne font que jouer le rôle ancestral qui leur est dévolu : la protection du peuple. Nous avons choisi ce document car les élèves peuvent le lire de façon réaliste selon les modalités dominantes en classe d'histoire (Audigier, 1995), le document dit le passé, est une description de ce qu'ont vécu les Vendéens. En
Voici ce que disent les élèves du groupe en étudiant le document des Mémoires de la noble:

Transcription de la séance 1, étape 2

\begin{tabular}{|c|c|c|}
\hline 93 & $\mathrm{E}$ & $\begin{array}{l}\text { Oui Monsieur Sapinaud de Bois-Huguet, oui } \\
\text { c'est ça. Mais c'est pas hyper objectif si tu fais } \\
\text { ça sur ton mari. Là elle a écrit sur son mari. }\end{array}$ \\
\hline 94 & A & Donc ça veut dire que les nobles étaient contre. \\
\hline 95 & $\mathrm{E}$ & $\begin{array}{l}\text { Ah voilà, et du coup, elle, était contre les } \\
\text { nobles, contre la guerre. }\end{array}$ \\
\hline 96 & A & $\begin{array}{l}\text { Ceux qui voulaient faire la guerre, c'était les } \\
\text { paysans. }\end{array}$ \\
\hline 97 & E & Oui regarde "une rébellion paysanne." \\
\hline
\end{tabular}


Puis la professeure intervient :

\begin{tabular}{|l|l|l|}
\hline 105 & A & $\begin{array}{l}\text { Là c'est la femme d'un noble, et le noble dit dans le texte que c'est impossible de faire la guerre, parce } \\
\text { que toute façon c'est perdu d'avance et que c'est les paysans qui veulent cette rébellion. }\end{array}$ \\
\hline 106 & Prof & $\begin{array}{l}\text { Commencer peut-être pour ce document par cette case-là : dans quel objectif ce texte a été écrit? Vous } \\
\text { avez compris qui c'est? }\end{array}$ \\
\hline 107 & E & Ben c'est les nobles mais pour dire au cas où qu'on les accuse, c'est pas eux qu'ont voulu la révolution. \\
\hline 108 & Prof & $\begin{array}{l}\text { Essayez de comprendre, regardez le titre, là, Mémoires. Quand on écrit ses mémoires, qu'est-ce que c'est } \\
\text { comme document historique ça? }\end{array}$ \\
\hline 109 & A & C'est pas objectif. \\
\hline 110 & E & C'est des preuves hein. \\
\hline 111 & Prof & $\begin{array}{l}\text { Des preuves? Si tu écris tes mémoires sur le voyage aux Pays-Bas, c'est une preuve de ce que tout le } \\
\text { monde a vécu? }\end{array}$ \\
\hline 112 & E & Ah non! Non non non! Justement c'est une... \\
\hline 113 & A & Son ressenti personnel! \\
\hline 114 & Prof & Un regard! Un ressenti. Donc commencez peut-être par cette case là pour ensuite revenir à l'autre. \\
\hline 115 & J & Oui ils mettent la faute sur les paysans. \\
\hline
\end{tabular}

Et les élèves concluent alors :

\begin{tabular}{|c|c|c|}
\hline 131 & $\mathrm{~J}$ & C'est vu comme si c'était des paysans qui avaient voulu faire cette guerre. \\
\hline 132 & E & $\begin{array}{l}\text { En gros les nobles, vu qu'ils recevaient de l'argent du roi, il fallait automatiquement qu'ils soient bien vus } \\
\text { par le roi. Ils allaient pas être contre le roi puisque ils avaient des privilèges. Alors que les paysans, eux, } \\
\text { qui n'avaient pas de privilèges, ils étaient forcément contre. }\end{array}$ \\
\hline 133 & A & Mais justement en Vendée, les paysans, ils sont pour le roi, c'est bizarre! \\
\hline 134 & $\mathrm{E}$ & Ah oui, ah mais oui. \\
\hline 135 & A & Donc c'est l'inverse, ils accusent, là c'est le contraire! \\
\hline 136 & E & Oui mais là c'est hyper compliqué dans ma tête. \\
\hline 137 & A & $\begin{array}{l}\text { En fait, genre, le roi donne des privilèges aux nobles. Donc c'est les nobles qui veulent que le roi reste, et } \\
\text { les paysans sont censés être contre le roi, et donc mener la Révolution normale. Pour moi là c'est inversé. }\end{array}$ \\
\hline 138 & E & Oui c'est pas logique. \\
\hline 139 & A & Du coup, là, ce serait un peu un mensonge. \\
\hline 140 & $\mathrm{E}$ & Non mais là y’a rien de logique dans l'histoire. \\
\hline 141 & $\mathrm{~J}$ & Mais c'est parce que ça a été écrit par un noble. C'est pour ça. \\
\hline 142 & $\mathrm{E}$ & $\begin{array}{l}\text { Madame, on a un gros problème de logique. Vu que les nobles... Pourquoi les nobles ils ne voulaient pas } \\
\text { faire... C'est l'inverse! }\end{array}$ \\
\hline 143 & A & $\begin{array}{l}\text { En fait, elle, ce qu'elle dit, c'est un mensonge, enfin, c'est son point de vue à elle. Elle, elle n'a aucune } \\
\text { raison de vouloir que le roi s'en aille! }\end{array}$ \\
\hline 144 & $\mathrm{E}$ & $\begin{array}{l}\text { Puisqu'elle a des privilèges. Alors que les paysans, eux, ils devraient vouloir que le roi s'en aille. } \\
\text { Sauf que non! }\end{array}$ \\
\hline
\end{tabular}

Dans les échanges ci-dessus ${ }^{4}$, on peut observer l'articulation des échelles entre le micro et le macro qui enclenche la problématisation historienne par la mise en tension entre les données et les modèles permettant une exploration des possibles. Entre les tours de parole 93 et 97, les élèves cherchent l'information qui permet de connaître le regard de cette noble sur les Guerres de Vendée sans lier la critique du document avec l'interprétation de son contenu. Ils sont ainsi capables de dire que cela ne doit pas 
être objectif, tout en conservant les dires de la noble tels quels, sans questionnements. Le basculement se fait au tdp 108, quand la professeure leur demande de s'interroger sur la nature du document, sur ce que sont des mémoires. Les élèves doivent articuler les échelles d'étude du document et ils considèrent celui-ci dans son ensemble, ce qui leur permet d'interpréter autrement les indices avec des hypothèses multiples. L'absence d'articulation des échelles de lecture du document micro et macro ne permet pas aux élèves de construire leur propre modèle explicatif car ils reprennent textuellement celui de l'auteure. L'articulation des échelles du document les pousse alors à s'interroger de façon historienne. Au tdp 105 et au tdp 143, la même élève explique ce qu'elle a compris du document. D'abord, sans articulation (tdp 105), elle ne lie pas la critique de la source avec son interprétation, elle sélectionne des éléments du document, des données, qu'elle organise en explication cohérente, des modèles. Mais ce sont les modèles de l'auteure et non pas ses propres idées explicatives. Avec l'articulation des échelles (tdp 143), un aller-retour est effectué entre la critique de la source et son interprétation, tout en tenant compte du savoir déjà-là (tdp 137). Elle prend du recul avec le document pour l'aborder dans son ensemble : un point de vue, un mensonge; cela permet ensuite aux élèves de réinterroger ce qui est dit dans le document, qui fait cas. Ils ne le croient plus : elle ne dit pas la vérité, mais alors pourquoi? Et la réponse ne peut pas se trouver seulement dans le document mais dans la construction de leurs propres modèles explicatifs et la mobilisation d'autres connaissances plutôt que dans la reprise de ceux de l'auteure. Le fait de fournir aux élèves le tableau n'a pas suffi pour que les élèves fassent ce changement d'échelle et l'intervention de la professeure a été nécessaire à celui-ci. C'est un point important de notre recherche. La problé- matisation peut difficilement se concevoir dans un dispositif d'ingénierie didactique figé dans un tableau pensé par le professeur. C'est le cadrage oral de celuici qui permet aux élèves novices de problématiser en les poussant à articuler les échelles du document.

\section{Prolonger l'articulation des échelles en allant plus loin sur la critique de la source}

Devant l'esquisse d'articulation des échelles du document par les élèves, nous décidons dans la séquence forcée, pour notre travail de chercheuse, de poursuivre ce travail. Suite à l'absence de réflexion des élèves sur la date et le contexte d'élaboration des documents, leur est distribuée lors de la deuxième séance une chronologie avec quelques indications : « Après la défaite de Napoléon en 1814, le frère de Louis XVI devient roi sous le nom de Louis XVIII. Il rétablit le drapeau blanc et « octroie » aux Français une Charte constitutionnelle. Quelques acquis de la Révolution sont reconnus mais le roi dispose de l'essentiel des pouvoirs. Ce n'est donc ni une démocratie, ni une monarchie absolue. » L'objectif pour prolonger le processus est de poursuivre ce qui a été mené en liant nature du document et interprétation pour qu'ils fassent de même avec la date de celui-ci et son contexte. Ils ont alors à expliquer ce que cela leur apporte pour l'analyse du document puis à revenir sur l'intention de la production de la trace. Voici le tableau à remplir et les échanges du groupe pour y parvenir :

\begin{tabular}{|l|l|}
\hline $\begin{array}{l}\text { Qu'est-ce qu'apporte l'éclai- } \\
\text { rage du contexte sur ce qui } \\
\text { est dit des Guerres de Vendée } \\
\text { dans ce document? }\end{array}$ & $\begin{array}{l}\text { Dans quel objectif a été } \\
\text { produite cette trace de }\end{array}$ \\
\hline & l'histoire? \\
\hline
\end{tabular}

Transcription de la séance 2, étape 3

\begin{tabular}{|c|c|c|}
\hline 108 & $\mathrm{~J}$ & $\begin{array}{l}\text { En fait, elle glorifie les paysans, sauf que elle prend du recul par rapport aux nobles, et elle dit que c'est } \\
\text { pas eux qui ont envie de faire la guerre c'est... }\end{array}$ \\
\hline 109 & A & $\begin{array}{l}\text { C'est pas eux qui sont à l'origine en fait. Elle se rend compte que ce n'est pas les nobles qui ont voulu } \\
\text { cette rébellion. }\end{array}$ \\
\hline 110 & $\mathrm{~J}$ & Ce qui est tout à fait un mensonge bien sûr. \\
\hline 111 & E & $\begin{array}{l}\text { Après, on avait mis : "écrit pour la transmission d'un point de vue sur cette période de l'histoire, celle } \\
\text { d'une noble. Pour éviter les accusations de leurs supérieurs, ils expliquent que cette rébellion est avant } \\
\text { tout paysanne." }\end{array}$ \\
\hline
\end{tabular}




\begin{tabular}{|c|c|c|}
\hline 112 & A & Beh du coup, c'est un point de vue noble... \\
\hline 113 & E & Sur cette époque. Après c'est ni une monarchie, ni une... c'est instable. \\
\hline 114 & A & Qui n'est ni une démocratie, ni une monarchie absolue. \\
\hline 115 & E & $\begin{array}{l}\text { Oui mais là, ça répond pas à la question dans quel objectif a été produite cette trace de l'histoire. } \\
\text { Pourquoi elle a écrit ça? Mis à part pour donner son point de vue? Quand ils écrivent ils veulent tous } \\
\text { donner leur point de vue mais là? }\end{array}$ \\
\hline 116 & J & $\begin{array}{l}\text { Elle dit que c'est les paysans qui ont voulu la guerre. À part la religion, ils devaient bien avoir une raison? } \\
\text { Le roi, la monarchie, quelque chose qui leur a été enlevé... Et pourquoi c'est que en Vendée? C'est pas } \\
\text { des paysans de Bretagne, c'est pas des paysans de Normandie, pourquoi en Vendée? Qu'est-ce qu'il y a } \\
\text { avait en Vendée pour que ce soit là? }\end{array}$ \\
\hline 117 & A & $\begin{array}{l}\text { On peut dire aussi leur acharnement parce que le mec là, Sapinaud, il essaie de les dissuader de partir et } \\
\text { au final la dernière phrase c'est "en voyant leur obstination, il finit par..." }\end{array}$ \\
\hline 118 & E & Ouai voilà. C'est qu'il ne le voulait pas vraiment. \\
\hline 119 & A & Ouai, c'est pour montrer l'obstination des Vendéens. \\
\hline 120 & $\mathrm{~J}$ & $\begin{array}{l}\text { J'ai une question, si c'est c'est les paysans qui ont voulu la guerre, quel serait leur intérêt? Pourquoi c'est } \\
\text { en Vendée qu'ils ont voulu la guerre et pas autre part? Est-ce qu'ils étaient plus riches, plus croyants ici? }\end{array}$ \\
\hline 121 & Prof & $\begin{array}{l}\text { Il y a une histoire de croyances en effet plus implantées en Vendée chez les paysans. Mais aussi on est } \\
\text { vraiment dans un espace où l'État est peu présent. On a l'image du roi mais les impacts de la Révolution, } \\
\text { ils arrivent très tardivement. }\end{array}$ \\
\hline 122 & $\mathrm{~J}$ & Spécifiquement en Vendée? \\
\hline 123 & Prof & $\begin{array}{l}\text { Pas que en Vendée, c'est dans les campagnes. Mais en fait, il n'y a pas qu'en Vendée qu'il y a des révoltes. } \\
\text { C'est que en Vendée, ça s'est installé. Parce qu'il y a eu aussi avec les nobles, ça s'est organisé d'une } \\
\text { certaine façon. Il n'y a pas qu'en Vendée qu'il y a des troubles, là on le retient mais il n'y a pas que là. La } \\
\text { Révolution qui démarre de Paris mais la France ne suit pas au même rythme. }\end{array}$ \\
\hline 124 & $\mathrm{~J}$ & $\begin{array}{l}\text { Merci. Bon, en fait ça explique tout maintenant. Oui c'est ça. Là on regarde à un endroit précis-là! Mais } \\
\text { c'est pas le seul qui a vécu... }\end{array}$ \\
\hline 125 & E & Non mais lisez le document 4. \\
\hline 126 & $\mathrm{~J}$ & $\begin{array}{l}\text { Alors pendant cette période-là, ça doit être celle-là. Oui c'est ça, c'est cette période où on glorifie le } \\
\text { régime du roi, 1817-1824, ils sont contre les révolutionnaires donc ils vont glorifier les guerres de } \\
\text { Vendée. Et on se concentre sur la Vendée pour la glorifier. }\end{array}$ \\
\hline
\end{tabular}

Dans ces échanges ${ }^{5}$, l'interaction micro-macro se confirme : les élèves mettent en rapport les données de la chronologie (la Restauration) avec ce que la noble dit. Cela leur permet une nouvelle exploration des possibles avec de questionnements nouveaux sur la période (tdp 116). Là où la réflexion sur les mémoires avait abouti au principe que la noble ment, cette contextualisation les fait réfléchir sur les raisons de mentir de façon plus précise (tdp 126) mais cela nécessite des connaissances supplémentaires : étaitce le seul espace à se rebeller? Ainsi, là où l'absence d'articulation des échelles du document focalisait les élèves sur ce que disait la noble, leur articulation a changé l'approche les faisant entrer dans l'exploration des possibles afin de comprendre les raisons qu'a l'auteure de produire ces explications.
Pour modéliser le processus de problématisation en histoire dans ces échanges, nous décidons d'utiliser un espace des contraintes (Orange 2005) qui schématise la modélisation en trois registres évoqués précédemment : celui des faits ou des données, celui des idées explicatives (ou des modèles), et le registre explicatif lui-même dans lequel fonctionnent les relations entre les deux premiers. Le premier est le registre empirique, celui des données sélectionnées par les élèves dans ce qu'ils savent déjà, ce qu'apporte le professeur ou ce qu'ils choisissent dans les documents, et qui contraint les réponses parce que la connaissance du passé repose sur les traces qu'il a laissées. Le registre des modèles est celui des modèles de comportements humains dont disposent les élèves pour rendre compte des actions des hommes. Il condi- 
tionne sur le plan théorique les réponses. La mise en tension entre des données et des modèles explicatifs en problématisation conduit à l'exploration de faits et de modèles alternatifs et à la production de conditions de pertinence de ces derniers. Enfin, les données mobilisées et les modèles explicatifs peuvent être regroupés dans des registres explicatifs qui sont les paradigmes que nous identifions plus haut et qui tiennent donc la cohérence du raisonnement.

\section{Espace des contraintes double problème en construction / articulation des échelles du document en histoire}

Nous avons décidé de faire évoluer l'espace des contraintes tel qu'il existe dans le cadre de la problématisation pour y inclure le passage à l'articulation des échelles du document, il s'agit de mêler l'événement étudié, les Guerres de Vendée et la façon des élèves de considérer ce que dit l'auteur du document et pourquoi elle le dit. Il faut donc qu'il puisse représenter les deux échelles d'analyse (micro et macro) et leurs interactions l'une sur l'autre, puisque nous considérons que c'est par l'articulation entre le macro et le micro que la problématisation est possible et permet davantage d'exploration.

À gauche, sont représentées les deux échelles, sans interactions et donc sans la mise en tension entre données et modèles puisque les élèves prélèvent des éléments du texte comme des informations venues du passé à ne pas soumettre à la critique. La présentation du document n'est alors pas utilisée pour l'interprétation. Les modèles explicatifs sont donc ceux de la noble qu'ils ne questionnent pas. Le registre explicatif (Rex) est ethnologique et le document vu comme réaliste. La noble produit ses explications en considérant le peuple vendéen de façon essentialisée, et les élèves ne questionnant pas cette logique, ils ne font pas un travail problématisé puisqu'il n'y a pas de mise en tension entre données et modèles explicatifs.

À droite, l'articulation des échelles est à l'œuvre avec une interaction micro-macro permise par le questionnement de l'intention de l'auteure, son statut, le point de vue qu'elle est censée avoir au regard des connaissances qu'ils ont sur la période. Cela produit une nécessité sur le problème en construction qui est qu'une telle subjectivité de l'auteure donne à penser qu'elle ment. C'est un aspect essentiel pour que les élèves entrent dans l'étude critique du document. Pour la professeure, comme pour les historiens, il n'existe pas de documents non subjectifs, alors que pour les élèves, cela semble être possible. Cette nécessité de penser le document comme subjectif est donc un préalable à l'articulation des échelles du document. Les élèves cherchent alors à répondre à l'intention de ce mensonge en revenant à l'échelle micro mise en tension avec l'échelle macro pour produire du savoir nouveau : si elle ment, quelles sont ses motivations? Ce registre explicatif est complexe car il oblige à considérer le document comme une trace du passé à soumettre à la critique historienne. Sont mis en tension les données issues de la critique de la source, les données fournies par l'auteur et les modèles produits par les élèves pour expliquer ce récit sur le passé. Cette problématisation effectuée avec l'aide de la professeure qui les pousse à mettre en relation critique de la source et interprétation du document produit du savoir qui fait sens au regard de l'épistémologie de l'histoire.

\section{Retour réflexif sur les sites touristiques}

En fin de séquence, les élèves doivent revenir en groupe sur le récit structuré dans les sites touristiques en Vendée :

\begin{tabular}{|l|l|l|}
\hline 1 & $\begin{array}{l}\text { Prof } \\
1 \text { h17 }\end{array}$ & $\begin{array}{l}\text { Ensuite, deuxième question, là ce sera } \\
\text { pour revenir à ce qu'on avait fait au } \\
\text { tout début, quelle vision des guerres } \\
\text { de Vendée est entretenue dans les sites } \\
\text { touristiques évoqués au tout début de la } \\
\text { séquence. On avait parlé du Puy du Fou, } \\
\text { certains m'avaient parlé de l'Historial, la } \\
\text { Chabotterie, etc.. C'est quelle vision dans } \\
\text { les regards qu'on a vue et qu'est-ce que } \\
\text { cela dit des relations entre ce territoire, } \\
\text { c'est à dire la Vendée, et cette histoire-là. } \\
\text { Ok? (...) }\end{array}$ \\
\hline
\end{tabular}

Nous choisissons de chercher les ponts qu'ils ont pu faire avec la problématisation effectuée à partir de l'étude du document sur les mémoires d'une noble. Les élèves du groupe reprennent notamment les éléments de glorification qu'ils avaient esquissés comme explication à la construction d'un récit sur les Guerres de Vendée. Ils confirment dans les échanges suivants la compréhension du paradigme dans lequel était la noble et sont aujourd'hui les sites touristiques $^{6}$ : 
Transcription de la séance 2, étape 5

\begin{tabular}{|c|c|c|}
\hline 23 & E & Au Mémorial de la Vendée, c’est vachement religieux. \\
\hline 24 & M & Ah ouais? \\
\hline 25 & E & $\begin{array}{l}\text { Ben, je sais pas, j’y ai été il y a hyper longtemps. Beh vu que eux ils étaient très religieux, c'est la version } \\
\text { que eux ils veulent, celle de la monarchie. }\end{array}$ \\
\hline 26 & $\mathrm{~J}$ & Oui la version monarchiste. \\
\hline 27 & E & La vision est religieuse... \\
\hline 28 & $\mathrm{~J}$ & Mais on a dit que c'était pas qu'en Vendée en fait. \\
\hline 29 & E & $\begin{array}{l}\text { Très monarchiste, étant donné... Qu'ils étaient pour le retour à la monarchie. Ils glorifient donc leurs } \\
\text { batailles face aux Républicains. Qu'est-ce que cela dit des relations de ce territoire et de son histoire? }\end{array}$ \\
\hline 30 & A & En gros, la Vendée et son histoire? C'est ça? \\
\hline 31 & E & Beh oui mais quoi? Madame, la dernière question, on comprend pas en fait. \\
\hline 32 & Prof & $\begin{array}{l}\text { Alors, faites voir ce que vous avez répondu avant? Alors aujourd'hui, il y a très peu de Vendéens qui } \\
\text { sont monarchistes, vous êtes d'accord, ça reste minoritaire. Mais n'empêche qu'il y a dans tout ce } \\
\text { qu'on met en scène, au Puy du Fou, à la Chabotterie et autres, un certain rapport à l'histoire. Et qui est } \\
\text { entretenu parce qu'on emmène les classes, les primaires vont le voir, au collège on va le voir. Donc on } \\
\text { entretient quelque chose dans la tête des Vendéens. C'est quoi justement le rapport entre ce territoire de } \\
\text { la Vendée et son histoire? }\end{array}$ \\
\hline 33 & $\mathrm{~J}$ & C'était pas que du bon la révolution, tout le monde n'était pas d'accord. \\
\hline 34 & Prof & $\begin{array}{l}\text { Si vous allez dans un musée à Nantes qui parle de la Révolution, on ne va pas vous en parler de la même } \\
\text { façon que si vous allez à l'Historial. Pas du tout et c'est l'inverse. }\end{array}$ \\
\hline 35 & M & Ça veut dire que les Vendéens cherchent en fait à laisser une trace pour... \\
\hline 36 & E & De leurs batailles? \\
\hline 37 & M & Ouais c'est ça. \\
\hline 38 & J & C'est aussi que pas tout le monde était d'accord avec la Révolution. \\
\hline 39 & A & $\begin{array}{l}\text { Moi je sais pas, mais quand j'ai été au Puy du Fou, j'avais l'impression que c'était une terre, un haut lieu } \\
\text { de... Alors, la Vendée a sa propre vision des guerres qu'elle a faite... }\end{array}$ \\
\hline 40 & E & Et souhaite entretenir la mémoire qui n’est pas la même que celle de toute la France. \\
\hline 41 & A & Elle souhaite entretenir... \\
\hline 42 & $\mathrm{E}$ & Un devoir de mémoire. \\
\hline
\end{tabular}

La conclusion des élèves permet de dépasser l'opposition des récits sur le passé dans lequel on peut facilement se placer quand on étudie le regard porté par les sites touristiques sur les Guerres de Vendée. Ils prennent en compte le registre explicatif ethnologique qui régit ces sites : le royalisme (tdp 25-26-29) comme porteur de valeurs politiques et ils reviennent vers les données qu'ils expliquent ainsi par une glorification de cette période (tdp 29), d'une vision différente de celle de la France qui implique, de par cette différence, un devoir de mémoire souhaitant être entretenu (tdp 40-42). Ainsi, les élèves n'ont pas à choisir s'il y a manipulation ou pas de l'histoire dans les sites touristiques sur les Guerres de Vendée mais ils problématisent sur les raisons pour ces sites de présenter une telle version des choses : le parc "souhaite entretenir la mémoire qui n'est pas la même que celle de toute la France ».

\section{CONCLUSION}

Dans les extraits de corpus étudiés, c'est l'absence d'articulation des échelles d'analyse qui empêchait les élèves d'aborder de façon historienne le document. Rester à l'échelle micro (les informations 
dans le document) ne leur donnait accès qu'aux modèles explicatifs mobilisés par l'auteure, une noble. L'échelle macro (les données contextuelles du document), bien qu'utilisée, n'était pas mise en relation avec ce qui était dit dans le texte. En étant contraints à l'articulation par le dispositif (tableau et cadrage oral de la professeure), ils ont considéré ces mémoires comme une trace et les informations transmises par la noble sont devenues des indices d'une enquête. Ils ont alors pu construire de nouveaux modèles explicatifs justifiés dans le groupe par l'exploration des possibles mettant en tension les données et les explications qu'ils avaient déjà proposées. Ces modèles sont ensuite repris pour réfléchir sur les discours produits par les sites touristiques, porteurs eux aussi d'un registre explicatif ethnologique. Plutôt que les contredire de façon frontale en classe d'histoire, ce que nous avons proposé est de partir d'une question socialement vive localement pour interroger les raisons de la production de tels récits sur le passé, dans des contextes particuliers.

Les élèves ont ainsi construit un problème historique sur les mémoires développées après un évènement. Ils ont fait de l'histoire dans le sens où ils se sont posé des questions sur les traces du passé plutôt que sur le passé lui-même, ce qui est le fondement même de cette discipline. L'adaptation que nous avons faite de l'espace des contraintes nous a permis de souligner la spécificité de la didactique de l'histoire dans le cadre théorique de la problématisation en montrant la nécessité de l'articulation des échelles du document pour explorer les possibles.

\section{NOTES}

1. Martin, J.-C. (2012). Vendée, où est le génocide? Causeur, 24/10/12

2. Les documents : Portrait d'Henri de La Rochejacquelein de Narcisse Guérin (1817), extrait des Mémoires de JeanneAmbroise de Sapinaud de Bois-Huguet (1824), extrait des Mémoires pour servir à l'histoire de la guerre de Vendée 1795 , peinture de la Mort de Joseph Bara de Charles MoreauVauthier (1880).

3. Séance 1 , étape 1 : Les élèves sont en groupes et doivent élaborer une hypothèse sur les regards portés sur les Guerres de Vendée après avoir évoqué les sites touristiques qui en parlent.
4. Séance 1 , étape 2 : Les élèves sont en groupe et doivent remplir le tableau expliqué plus haut.

5. Séance 2, étape 3 : Les élèves sont en groupe et doivent remplir un tableau qui aborde le contexte du document, après avoir réfléchi sur les explications des groupes lors de la séance précédente, fournies sous forme de transcriptions. 6. Séance 2 , étape 5 : après l'étape 4 de synthèse, les élèves doivent répondre en groupe à des questions lors de l'étape 5 pour expliquer les regards portés sur les Guerres de Vendée par les sites touristiques.

\section{RÉFÉRENCES}

Audigier, F. (1992). Documents, des moyens pour quelles fins? Actes du $7^{e}$ colloque. Paris : INRP.

Audigier, F. (1995). Histoire et géographie : des savoirs scolaires en question entre les définitions officielles et les constructions des élèves. Spirale - Revue de Recherches en Education, 15, 61-89.

Avezou, L. (2013). Les mythes de l'histoire de France. Paris : La Boetie.

Bazin, J. (2008). Des clous dans la Joconde. L'anthropologie autrement. Toulouse : Anacharsis.

Bloch, M. (1997). Apologie pour l'histoire ou Métier d'historien. Paris : Armand Colin (première édition, 1949).

Buisson, P. (2017). La grande histoire des Guerres de Vendée. Paris : Perrin.

Cariou, D. (2012). Historisation de la didactique de l'histoire : démarches de pensée historienne et apprentissage de l'histoire In Les didactiques en question(s). Etat des lieux et perspectives pour la recherche en formation. Bruxelles : De Boeck, 69-78.

Chartier, R. (1990/2000). Les origines culturelles de la Révolution française. Paris : Le Seuil, « Points ».

Doussot, S. (2010). Pratiques de savoir en classe et chez les historiens : une étude de cas au collège. Revue française de pédagogie, 173, 85-104.

Doussot, S. (2011). Didactique de l'histoire. Outils et pratiques de l'enquête historienne en classe. Rennes: PUR.

Doussot, S. \& Vézier, A. (2014). Des savoirs comme pratiques de problématisation : une approche sociocognitive en didactique de l'histoire. Éducation E Didactique, 8(3), 111-140.

Fabre, M. (2015). Éducation et humanisme. Lecture de John Dewey. Paris : Vrin.

Fabre, M. (2017). Qu'est-ce que problématiser? Paris : Vrin. Fink, N. (2014). Paroles de témoins, paroles d'élèves : La mémoire et l'histoire de la Seconde Guerre mondiale, de l'espace public au monde scolaire. Berne : Peter Lang.

Ginzburg, C. (2008). Préface. Preuves et possibilités. In N. Z. Davis, Le retour de Martin Guerre, 9-43. Paris : Tallandier (Première édition, Harvard University Press, 1983). 
Legardez, A. \& Simonneaux, L. (2006). L'école à l'épreuve de l'actualité - Enseigner les questions vives. Issy-lesmoulineaux : éd. ESF.

Le Marec, Y., Doussot, S. \&Vézier, A. (2009). Savoirs, problèmes et pratiques langagières en Histoire. Éducation E Didactique, 3(3), 7-27.

Letourneau, J. \& Moisan, S. (2004). Mémoire et récit de l'aventure historique du Québec chez les jeunes Québécois d'héritage canadien-français. The Canadian Historical Review, vol. 85, no 2, 2004, 325-356.

Martin, J-C. (1992). La Vendée dans l'histoire des droites, in Sirinelli, J-F \& Vigne, E. (dir.). Histoire des droites. (II, 437-470). Paris : Gallimard.

Martin, J-C. (2000). À propos du « génocide vendéen ». Du recours à la légitimité de l'historien. Sociétés contemporaines, 39, 23-38.

Martin, J.-C. (2016). Robespierre, la fabrication d'un monstre. Paris : Perrin.

Martin, J-C. \& Suaud, C. (1996). Le Puy du Fou. Paris : LHarmattan.

Orange, C. (2005). Problématisation et conceptualisation en sciences et dans les apprentissages scientifiques. Les sciences de l'éducation - Pour l'ère nouvelle, 38(3), 69-93.

Orange, C. (2010). Situations forcées, recherches didactiques et développement du métier enseignant. Recherches en éducation, HS(2), 73-85.

Prost, A. \& Winter, J. (2004). Penser la Grande Guerre. Paris : Le Seuil, « Points».

Sécher, R. (1986). Le génocide franco-français, la VendéeVengé. Paris : PUF.

Tutiaux-Guillon, N. (2008). Interpréter la stabilité d'une discipline scolaire : l'histoire-géographie dans le secondaire français. In Audigier, F. \& Tutiaux-Guillon, N. (dir.). Compétences et contenus. Les curriculums en questions. Bruxelles : De Boeck, 117-146. 